\title{
Superiority of cut-and-sew technique for the Cox maze procedure: Comparison with radiofrequency ablation
}

John M. Stulak, MD, Joseph A. Dearani, MD, Thoralf M. Sundt III, MD, Richard C. Daly, MD, Christopher G. A. McGregor, MD, Kenton J. Zehr, MD, and Hartzell V. Schaff, MD

From the Division of Cardiovascular Surgery, Mayo Clinic and Foundation, Rochester, Minn.

Hartzell Schaff and John Stulak report support to the Mayo Clinic Cardiovascular Surgery Laboratory from AtriCure and Medtronic.

Read at the Eighty-sixth Annual Meeting of The American Association for Thoracic Surgery, Philadelphia, Pa, April 29-May 3, 2006.

Received for publication May 3, 2006; revisions received Aug 21, 2006; accepted for publication Sept 8, 2006.

Address for reprints: Hartzell V. Schaff, MD, Division of Cardiovascular Surgery, Mayo Clinic College of Medicine, 200 First St SW, Rochester, MN 55905 (E-mail: schaff@mayo.edu).

J Thorac Cardiovasc Surg 2007;133:1023-7 $0022-5223 / \$ 32.00$

Copyright (๑) 2007 by The American Association for Thoracic Surgery

doi:10.1016/j.jtcvs.2006.09.115
Objective: Although radiofrequency ablation is increasingly used to create the atrial lesions of the Cox maze procedure, its effectiveness in ablating atrial fibrillation compared with the standard cut-and-sew method is not known. We compare the freedom from atrial fibrillation in patients undergoing both methods with identical lesion sets.

Methods: Radiofrequency ablation was used to create full Cox maze lesions in 56 patients between January 2002 and February 2005; these patients were matched with those who underwent the standard cut-and-sew method. Matched variables were gender (33 male, 23 female, both), age (67.5 vs 67.2 years), New York Heart Association class (mean 2.28 vs 1.96), atrial fibrillation type (37 paroxysmal, 19 continuous, both), and concomitant mitral valve surgery (37 in both). Hypertension, preoperative left atrial size, and preoperative duration of atrial fibrillation were similar between groups.

Results: When compared with matched controls, fewer patients undergoing radiofrequency ablation were free from atrial fibrillation at dismissal $(63 \%$ vs $88 \% ; P=$ $.0039)$ and at last follow-up (62\% vs $92 \% ; P=.016)$. According to logistic regression for matched pairs, patients undergoing radiofrequency ablation were 4.5 times more likely to be in atrial fibrillation at dismissal (95\% confidence intervals $[\mathrm{CI}], 1.8,10.9)$ and 5 times more likely to be in atrial fibrillation at follow-up (95\% CI, 1.4, 17.3). No other covariate was associated with atrial fibrillation status at hospital dismissal or follow-up.

Conclusion: Creating Cox maze lesions with radiofrequency ablation is associated with less freedom from atrial fibrillation both early and late postoperatively. Because transmurality can be assured, the standard cut-and-sew Cox maze procedure remains the gold standard for the surgical treatment of atrial fibrillation.

$\mathrm{T}$ he classic cut-and-sew (CS) Cox maze III procedure is widely established as effective for the surgical treatment of atrial fibrillation (AF). However, its widespread adoption has been inhibited by its technical complexity. Alternate energy sources and alternate lesion sets have been proposed and used clinically in the interest of simplifying the procedure. ${ }^{1-4}$ Both on-pump and off-pump mazelike procedures have been described with epicardial as well as endocardial application of energy. ${ }^{5,6}$ The impact of these modifications on the effectiveness of curing $\mathrm{AF}$, however, remains unclear.

The largest clinical experience using alternate energy sources is with radiofrequency (RF) ablation, which uses alternating current to transfer energy to atrial tissue. Success of this technology in the catheterization laboratory has led surgeons to apply RF directly to the heart during cardiac surgery. ${ }^{7-9}$ Several instruments have been developed to create atrial lesions, including rigid unipolar probes with cooled tips, ${ }^{10,11}$ flexible unipolar probes, ${ }^{12}$ and bipolar clamps with $^{13}$ and without irrigation. $^{14}$ 


$$
\begin{aligned}
& \text { Abbreviations and Acronyms } \\
& \begin{aligned}
\mathrm{AF} & =\text { atrial fibrillation } \\
\mathrm{CI} & =\text { confidence interval } \\
\mathrm{CS} & =\text { cut-and-sew } \\
\mathrm{NYHA} & =\text { New York Heart Association } \\
\mathrm{RF} & =\text { radiofrequency }
\end{aligned}
\end{aligned}
$$

Despite reports of successful ablation of AF with these new instruments and techniques, the effectiveness of RF compared with the standard CS maze procedure has not been clearly demonstrated. Analysis of results is further complicated by variable definitions of success. We sought to more precisely evaluate the impact of one ablation technology by direct comparison with the CS technique using a case-matched study design holding the lesion set constant and evaluating cure by several clearly defined criteria.

\section{Patients and Methods}

From January 2002 to February 2005, 56 patients underwent creation of the full atrial lesion sets as described by Cox and associates ${ }^{15}$ using bipolar RF ablation. The pulmonary veins were ablated in identical fashion to the manner in which the pulmonary vein-encircling incision is created in the CS maze, and RF was used to create the connecting lesion to the mitral valve. Cryolesions were placed at both the mitral and tricuspid valve annuli, as in the CS maze. The transseptal lesion was created with a line of cryolesions to avoid injury to the blood supply to the sinoatrial node. We use this modification as part of our lesion set for the CS maze. The lesion sets were identical between the RF and CS groups, as were the placement of cryolesions. The only difference between the two procedures was in the method of creating the atrial lesions.

These patients were strictly matched with 56 patients from a database of 335 patients who underwent the classic CS Cox maze procedure at our institution between March 1993 and December 2002. Medical records were reviewed for patient demographics, past cardiac medical and surgical history, operative procedure, preoperative and postoperative cardiac rhythm, early and late morbidity, and survival.

Matched variables were gender, age, New York Heart Association (NYHA) class, AF type, and concomitant mitral valve as

TABLE 1. Matched variables between treatment groups

\begin{tabular}{lccc}
\hline Variable & $\mathbf{R F}$ & $\mathbf{C S}$ & $\boldsymbol{P}$ value \\
\hline Male & $33(59 \%)$ & $33(59 \%)$ & \\
Age range $(\mathrm{y})$ & $39-84$ & $38-80$ & \\
Median age $(y)$ & 67.5 & 67.2 & .87 \\
NYHA class & 2.28 & 1.96 & .07 \\
Chronic AF & $37(66 \%)$ & $37(66 \%)$ & \\
MV surgery & $37(66 \%)$ & $37(66 \%)$ & \\
\hline
\end{tabular}

RF, Radiofrequency maze group; $C S$, cut-and-sew maze group; NYHA, New York Heart Association; $A F$, atrial fibrillation/flutter; $M V$, mitral valve. shown in Table 1. Additional variables potentially affecting outcome, including hypertension (RF vs CS) (23 vs 22 patients; $P=$ $.85)$, left atrial size (67.5 vs $66.7 \mathrm{~mm} ; P=.799)$, and preoperative duration of $\mathrm{AF}(52 \pm 10$ vs $67 \pm 4$ months; $P=.69)$, were similar between groups.

\section{Statistical Analysis}

Demographic and other patient-related data were obtained from Mayo Clinic medical records. Follow-up information was obtained from subsequent clinic visits, written correspondence from local physicians, and mailed questionnaires to patients or families. Rhythm was evaluated by electrocardiograms obtained during clinical follow-up. Data were expressed as both median with a range and mean \pm standard deviation. A multivariable analysis (logistic regression for matched pairs) was used to identify factors for AF recurrence (hospital dismissal and last follow-up), normal sinus rhythm status (hospital dismissal and last follow-up), and need for postoperative permanent pacemaker. A KaplanMeier curve was generated to delineate time-related recurrence of AF. Early operative mortality was defined as death occurring within 30 days of operation or at any time during the index hospitalization. The Mayo Foundation Institutional Review Board approved this study, and all patients or their families gave written informed consent.

\section{Results}

Operative details are shown in Table 2. A classic Cox maze procedure was performed in all 56 patients with bipolar RF ablation (AtriCure device [AtriCure, Inc, Cincinnati, Ohio] in 50; Medtronic device [Medtronic, Inc, Minneapolis, Minn] in 6) to create the full Cox maze lesion sets. Six different surgeons performed the RF maze procedure on these 56 patients, and these same surgeons performed the CS maze as well. All patients had concomitant procedures performed, with more tricuspid valve interventions in the RF group $(P=.02)$. There was a trend toward more aortic valve procedures and septal myectomies among the RF group as well. Mean crossclamp time was similar between treatment groups (RF vs CS) $(65 \pm 6$ minutes vs $71 \pm 4$ minutes; $P=.6$ ), as was total bypass time (98 \pm 6 minutes vs $101 \pm 7$ minutes; $P=.54)$.

TABLE 2. Concomitant procedures performed

\begin{tabular}{lccc}
\hline Procedure & RF & CS & $P$ value \\
\hline TV surgery & $14(25 \%)$ & $5(9 \%)$ & .02 \\
AV surgery & $11(20 \%)$ & $4(7 \%)$ & .05 \\
CABG & $13(23 \%)$ & $11(20 \%)$ & .63 \\
Septal myectomy & $6(11 \%)$ & $1(2 \%)$ & .05 \\
MV surgery & $37(66 \%)$ & $37(66 \%)$ & 1.0 \\
\hline
\end{tabular}

$R F$, Radiofrequency maze group; $C S$, cut-and-sew maze group; $T V$, tricuspid valve; $A V$, aortic valve; $C A B G$, coronary artery bypass grafting; $M V$, mitral valve. 
TABLE 3. Freedom from AF according to three different methods of reporting

\begin{tabular}{|c|c|c|c|c|c|c|c|}
\hline \multirow[b]{2}{*}{ Group } & \multirow[b]{2}{*}{ Last FU } & \multicolumn{3}{|c|}{ Interval contact } & \multicolumn{3}{|c|}{ Actuarial } \\
\hline & & $6 \mathrm{mo}$ & $12 \mathrm{mo}$ & $15 \mathrm{mo}$ & $6 \mathrm{mo}$ & $12 \mathrm{mo}$ & $15 \mathrm{mo}$ \\
\hline RF & $62 \%$ & $75 \%$ & $62 \%$ & $50 \%$ & $87 \%$ & $76 \%$ & $61 \%$ \\
\hline CS & $92 \%$ & $91 \%$ & $91 \%$ & $91 \%$ & $94 \%$ & $91 \%$ & $91 \%$ \\
\hline
\end{tabular}

$\overline{A F}$, Atrial fibrillation; $R F$, radiofrequency ablation group; $C S$, cut-and-sew maze group; $F U$, follow-up.

There were no early deaths in either group. Early nonfatal morbidity in the RF group included postoperative $\mathrm{AF}$ ( $\mathrm{n}=24,43 \%$ of early survivors), cerebrovascular accident $(n=2)$, and ventricular arrhythmias, respiratory insufficiency, and complete heart block in 1 patient each. In the CS group, postoperative AF occurred in $24 \%(\mathrm{n}=13)$ of early survivors $(P<.05$ vs RF group), and cerebrovascular accident, ventricular arrhythmias, and respiratory insufficiency occurred in 1 patient each. Reoperation for mediastinal bleeding was required in $2 \mathrm{RF}$ patients and none of the CS patients. Two RF patients required reoperation for postoperative mitral valve $(\mathrm{n}=1)$ and tricuspid valve regurgitation $(\mathrm{n}=1)$.

Freedom from AF at dismissal was significantly lower in the RF group than in the CS group (64\% vs $88 \% ; P=$ .0039). According to a multivariable model, patients in the RF group were 4.5 times more likely to be in AF at hospital dismissal. The use of RF ablation was the only significant predictor of AF status at dismissal (RF, $P=.011$; tricuspid valve surgery, $P=.6031$; aortic valve surgery, $P=.4643$; septal myectomy, $P=.8184$ ).

New permanent pacemaker implantation was required in significantly more patients in the RF group than in the CS group (25\% vs $5 \% ; P=.004)$. In the RF group, indications for permanent pacemaker implantation were sinus node dysfunction in 12 patients, long QT syndrome in 1, and complete heart block in 1 . In the CS group, permanent pacemaker implantation was required for sinus node dysfunction in all 3 patients. According to a multivariable model, patients in the RF group were 4 times more likely to be dismissed with a new permanent pacemaker $(P=.02)$.

Of the 56 early survivors, 8 were lost to follow-up in the RF group and 2 in the CS group. Late follow-up extended up to 33 months (mean 8.4 months, median 8 months). Follow-up results of these 48 patients were then compared with their respective 48 matched controls from the CS group. The CS control matches for the 8 RF patients lost to follow-up were excluded from comparison. Although the CS maze patients had a longer follow-up period, "rhythm at last follow-up" was defined as last follow-up for the RF maze patients and their respective matches from the CS group at the same period in time postoperatively. In addition, when RF patients ceased follow-up, their respective

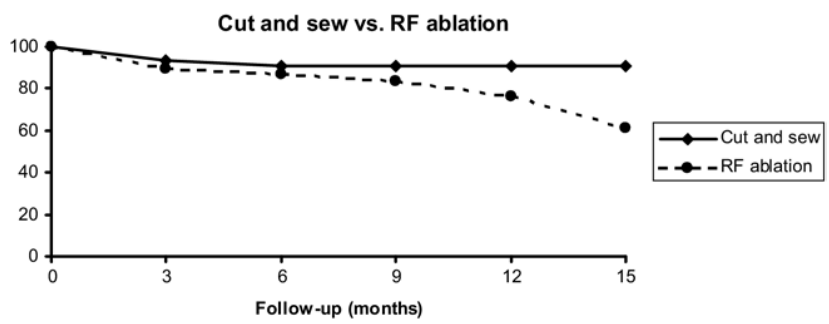

Figure 1. Actuarial freedom from AF between the RF and CS maze groups. Significance $(P<.05)$ was detected at 12 and 15 months between treatment groups. $A F$, atrial fibrillation; $R F$, radiofrequency ablation group; $C S$, cut-and-sew.

CS matches were excluded from analysis from that point onward. Patients available for follow-up in the RF group included 48 at 3 months, 26 at 6 months, 18 at 9 months, 12 at 12 months, and 12 at 15 months. There were 2 late deaths in the RF group resulting from noncardiac causes (malignancy in 1 and pneumonia in 1). Both patients were in AF before death. There were no late deaths in the CS group.

Antiarrhythmic medications at last follow-up were required in $36(75 \%)$ patients in the RF ablation group and 12 $(25 \%)$ patients in the CS control group $(P<.05)$. Forty-one $(85 \%)$ patients in the RF ablation group were still receiving warfarin anticoagulation at last follow-up compared with 12 (25\%) patients in the CS control group $(P<0.05)$.

Success of the procedure in treating AF was evaluated as rhythm at last follow-up, by actuarial methods, and by rhythm at interval contact (Table 3). Freedom from AF at last follow-up was significantly lower in the RF ablation group than in the CS control group (62\% vs $92 \% ; P=$ .0157). According to a multivariable model, RF ablation patients were 5 times more likely to be in $\mathrm{AF}$ at follow-up (95\% confidence intervals [CI]: 1.447, 17.267), and no covariate other than use of RF ablation was associated with presence of AF at last follow-up. Similarly, sinus rhythm at last follow-up was significantly less common in the RF ablation group than in the CS control group (43\% vs $85 \%$; $P=.00002$ ). According to a multivariable model, CS ablation patients were 10.5 times more likely to be in normal sinus rhythm at follow-up (95\% CI: 2.46, 45.45), and no covariate other than the use of RF ablation was associated with a decreased rate of sinus rhythm at last follow-up.

Use of a Kaplan-Meier curve to delineate time-related events shows that freedom from AF was $91 \%$ for the CS maze control group versus $87 \%$ for the RF ablation group at 6 months and $91 \%$ for the CS maze control group versus $76 \%$ for the RF ablation group $(P<.05)$ at 12 months (Figure 1). Reporting outcome as rhythm at 6-, 12-, and 15-month interval follow-up periods, freedom from AF was $91 \%$ for the CS maze control group versus $75 \%$ for the RF 


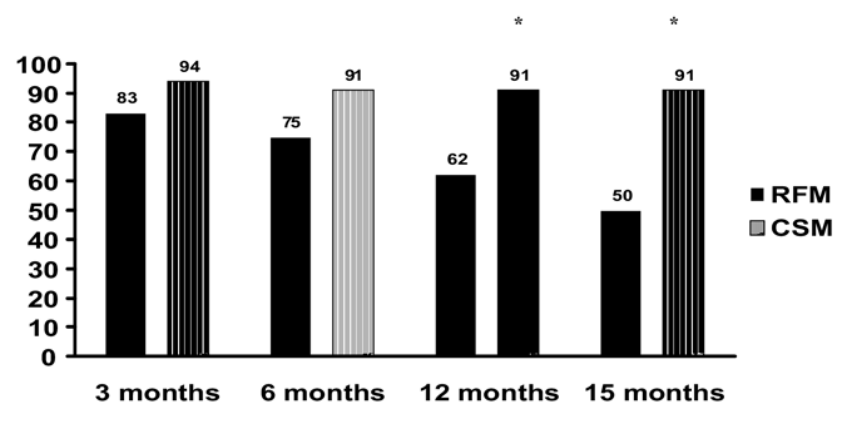

Figure 2. Freedom from AF at interval contact between the RF and CS maze (RFM and CSM) groups. Significance $(P<.05)$ was detected at 12 and 15 months between treatment groups. $A F$, atrial fibrillation; $R F$, radiofrequency ablation group; $C S$, cut-and-sew.

ablation group at 6 months, 91\% for the CS maze control group versus $62 \%$ for the RF ablation group $(P<.05)$ at 12 months, and $91 \%$ for the CS group versus $50 \%(P<.05)$ for the ablation group at 15 months (Figure 2).

\section{Conclusions}

In this study, the use of RF energy for the creation of the atrial lesions of the Cox maze procedure was associated with significantly less freedom from AF both at hospital dismissal and at follow-up as defined by rhythm at last follow-up, actuarial freedom from $\mathrm{AF}$, and rhythm at interval follow-up. Importantly, although this trend was consistent, the reported "success" of the procedure as defined by each of these methods differs. Patients in the RF ablation group were 4.5 times more likely to be dismissed from the hospital in AF and 5 times more likely to be in AF at the time of last follow-up. In a multivariable model, the only predictor for AF status at hospital dismissal and follow-up was the use of RF ablation at the time of the maze procedure. Further, patients in the CS cohort were twice as likely to be in sinus rhythm at hospital dismissal and 10.5 times more likely to be in sinus rhythm at follow-up. A recent meta-analysis of patients undergoing both the classic CS maze procedure and alternate energy sources demonstrates an $85.3 \%$ postoperative sinus rhythm rate for CS versus a $79.7 \%$ sinus rhythm rate when alternate energy is used. ${ }^{16}$ The current study demonstrates a much higher discrepancy between the two techniques.

A surprising finding of this study was that new permanent pacemaker implantation was required in significantly more patients in the RF group than in the CS group. Although the RF group underwent more tricuspid valve operations, as well as aortic valve replacements and septal myectomies, by multivariate analysis these were not significant predictors of new pacemaker implantation. Complete heart block would have been the expected indication for permanent pacemaker implantation if increased tricuspid valve surgery, aortic valve surgery, or septal myectomy placed the patient at higher risk for permanent pacemaker need. However, in these patients, the major indication for permanent pacemaker implantation was sick sinus syndrome. Perhaps the patients in the RF group represented a group with greater underlying cardiac disease (as evidenced by more concomitant procedures performed), and thus a higher incidence of sick sinus syndrome when AF was ablated.

Prior studies have documented that AF can be surgically ablated by RF energy. ${ }^{17-20}$ The comparative effectiveness with conventional surgical lesions, however, has not been previously subjected to case-matched comparison. There are theoretical reasons why these new RF ablation technologies may be less effective. Achieving and ensuring transmurality of lesions may be complicated by differences in atrial tissue characteristics (thickness, fibrosis, fat) as well as operative techniques (normothermia vs hypothermia, beating heart vs arrested heart). The unpredictability of conditions at the time of ablation will undoubtedly affect the quality of the lesions regardless of the energy source used. It is impossible to apply uniform energy and uniform techniques to atria that offer considerable variability. We continue to use cryolesions at the mitral and tricuspid valve annuli and to create the transseptal lesion, similar to our approach in the standard CS procedure. A different algorithm was used with the AtriCure device using two ablations to satisfactorily achieve a transmural lesion. We do not routinely evaluate transmurality by measuring electrophysiologic isolation of the pulmonary veins at the time of ablation, and there remains a question as to whether this equates with transmurality. Conversely, ablation procedures have been reported to convey high rates of success despite a demonstrably or even intentionally incomplete pulmonary vein encircling lesion. ${ }^{21}$

The literature on surgical treatment options for AF is complicated by multiple criteria for defining success. In our study, we report rhythm at last follow-up, actuarial freedom from AF, and rhythm at interval contact. In all instances, the results with CS were superior to RF, but the apparent "cure" rate differed among methods. These data highlight some of the difficulties in comparing reports of series of patients undergoing the Cox maze procedure or any other treatment for AF. It is therefore critical that criteria be clearly defined and that results be reported and analyzed in a uniform manner.

The retrospective, nonrandomized nature of this study is a major limitation. Although the groups were matched for demographics and clinical characteristics, there were differences in concomitant procedures performed that may have affected outcome. Additionally, although we report success in three ways, only electrocardiograms were analyzed and Holter monitoring was not performed. A significant percentage of AF may be entirely asymptomatic. ${ }^{22}$ In addition, 
although follow-up was also case-matched (matched controls were censored when RF patients ceased follow-up), there were a significant number of patients who lacked long-term follow-up. To account for this, we also reported success in a Kaplan-Meier analysis. The RF group represents the institution's first 56 patients in whom RF was used to create the maze lesion set. Individual surgeon learning curve, as well as a potential institutional learning curve, may have influenced our results when compared with other surgical series using RF. However, analysis of individual surgeon outcome, as well as analyzing the first group of 28 procedures and the second group of 28 procedures performed, did not yield any significant differences in outcome. Despite these shortcomings, the principal strength of this study is the consistency in the lesion set, leaving only lesion creation source as a variable.

The underlying mechanism for the observed difference in results may or may not be completely attributable to a lack of transmurality, but comparative studies such as this one raises important issues for consideration for future studies. Most important, the discrepancy in reported results for ablation tools is most likely multifactorial (differing techniques, differing methods of assessment and reporting success) and the ultimate clinical impact of transmurality and selection of lesion set is not completely understood.* More comparative studies are required to compare not only the efficacy of products from different companies, but also different alternate energy sources used. The ultimate goal in addressing these issues is to develop more successful ablation procedures.

If the indication for operation is medically refractory $\mathrm{AF}$, the CS Cox maze operation offers the patient the highest chance at freedom from AF. However, the use of RF while performing concomitant operations may simplify the procedure significantly but at the expense of a potentially lower chance of ablation of AF.

\section{References}

1. Kottkamp H, Hindricks G, Hammel D, Autschbach R, Mergenthaler J, Borggrefe $\mathrm{M}$, et al. Intraoperative radiofrequency ablation of chronic atrial fibrillation: a left atrial curative approach by elimination of anatomic "anchor" reentrant circuits. J Cardiovasc Electrophysiol. 1999;10:772-80.

2. Chen MC, Guo GB, Chang JP, Yeh KH, Fu M. Radiofrequency and cryoablation of atrial fibrillation in patients undergoing valvular operations. Ann Thorac Surg. 1998;65:1666-72.

3. Hemmer W, Botha C, Ickrath O, Starck C, Paula J, Roser D, et al. Background and early results of a modified left atrial radiofrequency procedure concomitant with cardiac surgery. Cardiovasc $J$ S Afr. 2001;12:19-28.

4. Pasic M, Bergs P, Muller P, Hofmann M, Grauhan O, Kuppe H, et al. Intraoperative radiofrequency maze ablation for atrial fibrillation: the Berlin modification. Ann Thorac Surg. 2001;72:1484-91.

5. Benussi S, Nascimbene S, Agricola E, Calori G, Calvi S, Caldarola A, et al. Surgical ablation of atrial fibrillation using the epicardial radio-

*Accord RD, Khargi K, Maessen JG. The issue of transmurality in surgical ablation for atrial fibrillation. Article in Cardiothoracic Surgery Network. 2006. frequency approach: mid-term results and risk analysis. Ann Thorac Surg. 2002;74:1050-6.

6. Melo J, Andragao P, Neves J, Ferreira M, Timoteo A, Santiago T, et al. Endocardial and epicardial radiofrequency ablation in the treatment of atrial fibrillation with a new intraoperative device. Eur J Cardiothorac Surg. 2000;18:182-6.

7. Haissaguerre M, Jais P, Shah DC, Gencel L, Pradeau V, Garrigues S, et al. Right and left atrial radiofrequency catheter therapy of paroxysmal atrial fibrillation. J Cardiovasc Electrophysiol. 1996;7:1132-44.

8. Chen SA, Hsieh MH, Tai CT, Tsai CF, Prakash VS, Yu WC, et al. Initiation of atrial fibrillation by ectopic beats originating from the pulmonary veins: electrophysiological characteristics, pharmacological responses, and effects of radiofrequency ablation. Circulation. 1999;100:1879-86.

9. Gaita F, Riccardi R, Calo L, Scaglione M, Garberoglio L, Antolini R, et al. Atrial mapping and radiofrequency catheter ablation in patients with idiopathic atrial fibrillation. Electrophysiological findings and ablation results. Circulation. 1998;97:2136-45.

10. Sie HT, Beukema WP, Misier AR, Elvan A, Ennema JJ, Haalebos $\mathrm{MM}$, et al. Radiofrequency modified maze in patients with atrial fibrillation undergoing concomitant cardiac surgery. J Thorac Cardiovasc Surg. 2001;122:249-56.

11. Sie HT, Beukema WP, Ramdat Misier AR, Elvan A, Ennema JJ, Wellens HJ. The radiofrequency modified maze procedure. A less invasive surgical approach to atrial fibrillation during open-heart surgery. Eur J Cardiothorac Surg. 2001;19:443-7.

12. Caccitolo JA, Stulak JM, Schaff HV, Francischelli D, Jensen DN, Mehra R. Open-heart endocardial radiofrequency ablation: an alternative to incisions in Maze surgery. J Surg Res. 2001;97:27-33.

13. Hamner CE, Potter DD Jr, Cho KR, Lutterman A, Francischelli D, Sundt TM 3rd, et al. Irrigated radiofrequency ablation with transmurality feedback reliably produces Cox-maze lesions in vivo. Ann Thorac Surg. 2005;80:2263-70.

14. Shimoike E, Kaji Y, Ueda N, Maruyama T, Kanaya S, Niho Y. In vivo and in vitro study of radiofrequency application with a new long linear probe: implication for the maze operation. J Thorac Cardiovasc Surg. 2000;120:164-72.

15. Cox JL, Jaquiss RDB, Scheussler RB, Boineau JP. Modification of the maze procedure for atrial flutter and atrial fibrillation. II. Surgical technique of the maze III procedure. J Thorac Cardiovasc Surg. 1995;110:485-95.

16. Khargi K, Hutten BA, Lemke B, Deneke T. Surgical management of atrial fibrillation: a systematic review. Eur J Cardiothorac Surg. 2005; 27:258-65.

17. Sie HT, Beukema WP, Elvan A, Ramdat Misier AR. Long-term results of irrigated radiofrequency modified maze procedure in 200 patients with concomitant cardiac surgery: six years' experience. Ann Thorac Surg. 2004;77:512-7.

18. Mohr FW, Fabricius A, Falk V, Autschbach R, Doll N, Von Oppell U, et al. Curative treatment of atrial fibrillation with intraoperative radiofrequency ablation: short term and midterm results. J Thorac Cardiovasc Surg. 2002;123:919-27.

19. Gillinov AM, McCarthy PM, Blackstone EH, Rajeswaran J, Pettersson G, Sabik JF, et al. Surgical ablation of atrial fibrillation with bipolar radiofrequency as the primary modality. $J$ Thorac Cardiovasc Surg. 2005;129:1322-29.

20. Gaynor SL, Diodato MD, Prasad SM, Ishii Y, Schuessler RB, Bailey MS, et al. A prospective, single-center clinical trial of a modified Cox maze procedure with bipolar radiofrequency ablation. $J$ Thorac Cardiovasc Surg. 2004;128:535-42.

21. van Brakel TJ, Bolotin G, Nifong LW, Dekker AL, Allessie MA, Chitwood WR Jr, et al. Robot-assisted epicardial ablation of the pulmonary veins: is a completed isolation necessary? Eur Heart J. 2005;26:1321-6.

22. Hindricks G, Piorkowski C, Tanner H, Kobza R, Gerds-Li JH, Carbucicchio C, et al. Perception of atrial fibrillation before and after radiofrequency catheter ablation: relevance of asymptomatic arrhythmia recurrence. Circulation. 2005;112:307-13. 


\section{Discussion}

Dr Cliff K. Choong (Cambridge, United Kingdom). Among the patients who underwent the RF ablation, did you and your coinvestigators do any intraoperative assessment to confirm transmurality of the ablation lines at all? Second, why do you think there is a difference in these results between the two groups? Last, on the basis of your findings, how has that influenced the practice at the Mayo Clinic?

Dr Stulak. Intraoperatively, it seemed in this patient population that RF was used mostly in patients who had numerous concomitant procedures. Because it is very time-consuming, we do not measure conduction block intraoperatively. It is hard to know why we saw the striking differences between the two procedures that we did. It is hard to know whether it is purely due to a potential lack of transmurality. There are numerous factors that may contribute, including differences in the atrial characteristics in terms of thickness, fibrotic scar in older patients, the amount of fat around the pulmonary veins, as well as intraoperative factors such as normothermia versus hypothermia, beating heart, endocardial versus epicardial. We think that it is impossible to apply uniform energy in a uniform fashion to atria that have very different characteristics. In our practice, although we believe that RF can be used as part of a concomitant procedure, if the indication for operation is purely AF, CS offers the patient the best relief of AF.

Dr Niv Ad. (Falls Church, Va). It was a wonderful paper. However, I think we should be very careful in interpreting the results, and this is why. By matching the patients, I think you made a crucial mistake. We all know that one of the most important and significant factors contributing to the failure of the maze procedure is the duration of AF before surgery. I did not see any matching between these two very significant parameters; that is, the type of $\mathrm{AF}$ was never found as a significant factor in predicting $\mathrm{AF}$ done in follow-up.

The other question that I have is more from a technical aspect. How can you perform the full maze procedure with a bipolar RF ablation technique? Can you elaborate more about this? And how many times do you clamp and ablate with the RF technique?

Last, it is kind of surprising to find such a stark difference between the two RF devices. Can you share with us your thoughts about why it happens?

Dr Stulak. Dr Ad, I appreciate your questions. It is difficult at times to perform the full Cox maze operation. The lesion sets are as close as they can be. The connecting lesion down to the mitral annulus from the encircling pulmonary vein lesion is the hardest one, and a lot of times the surgeons do what they can, but then use cryolesions as part of this lesion.

Dr Ad. Did you use cryolesions or not?

Dr Stulak. We did, at the mitral annulus and then at the tricuspid annulus, as described. We did our best to make the connecting lesion to the mitral annulus.

In terms of number of burns, with the AtriCure device, typically the surgeons at the Mayo Clinic apply two burns with each lesion set.

Dr Ad. What about the duration of AF before surgery? That is the most important factor in predicting failure.
Dr Stulak. We did not identify that in our biatrial experience to be a predictor of failure, only left atrial size and the presence of concomitant mitral valve surgery. In this study, patients in the CS group actually had a longer preoperative duration of their arrhythmia, which was not significant. This clinical characteristic did not seem to have any impact in this study, nor was that found to be significant in our biatrial CS experience. This is an important point that I failed to include in my presentation.

Dr Shelly C. Lall (St Louis, Mo). I would like to applaud you, Dr Stulak, and your colleagues for completing this study and presenting your data today. I have one question for you. Did the same surgeon that performed the CS procedures also do the RF ablation procedures? If not, could that have influenced your results?

Dr Stulak. Dr Lall, I enjoyed your presentation yesterday very much and I thank you for your question. There were six different surgeons performing the RF maze procedure, and these same six surgeons also performed the CS maze operations. I grant you, the patients in this series represent the first group of patients on whom we have used this technology, so of course, the question of a surgeon or even an institutional learning curve becomes relevant. We examined our results in terms of the surgeons and examined whether there were different freedom from AF rates for each, and there was no difference.

Dr Weisel. But the same surgeon did both procedures? That was her question.

Dr Stulak. All the surgeons at the Mayo Clinic have performed both operations.

Dr John Pepper (London, United Kingdom). Did you examine atrial transport or the pattern of ventricular filling and compare it with the two groups?

Dr Stulak. No, sir. That is very important. We did not. We strictly looked at freedom from AF.

Dr Pepper. Getting a nice pretty electrocardiogram is one thing, but altering atrial transport is another.

Dr Stulak. Point very well taken.

Dr John D. Puskas (Atlanta, Ga). Dr Stulak, the issues of transmurality are foremost in our minds. Your institution has provided some of the data demonstrating that transmurality can be very reliably achieved with bipolar RF. If we are achieving transmurality with bipolar RF, and we have to assume we are achieving transmurality with a CS technique, why is there a difference in these outcomes? Are we now to go back and doubt or question our transmurality data with either technique, or is it something different about the healing mechanism after an RF injury versus a CS injury, or is this simply some sort of an anomaly and we are not to draw any conclusion about the efficacy of RF?

Dr Stulak. Outstanding question. I think that the key is transmurality. Studies have shown that conduction block during surgery does not necessarily equate with transmurality and also that transmurality histologically does not equate with clinical success. I think we do not have a complete understanding of what is at play. Some studies have shown that transmurality cannot be achieved until some weeks or months after the operation. I think there is a lot about "transmurality" that we don't understand. 